\title{
Promoting Wellness through Electronic Home-Based Monitoring for Medically "At Risk" Elderly Persons
}

\author{
Thomas Falen, DHSc, MA, RHIA ${ }^{1,2}$, Alice M. Noblin, PhD, RHIA, CCS, PMP ${ }^{1}$
}

${ }^{1}$ Department of Health Management and Informatics, University of Central Florida, Orlando

${ }^{2}$ Arizona School of Health Sciences, A.T. Still University, Mesa, Arizona

Thomas.Falen@ucf.edu; Alice.Noblin@ucf.edu

*Corresponding Author: Thomas Falen, DHSC, MA, RHIA, Department of Health Management and Informatics, University of Central Florida, Orlando.

\begin{abstract}
There are growing numbers of medically "at-risk" elderly persons with chronic diseases, often living alone, who have difficulty managing their health and wellness on a daily basis. While elderly persons who are medically "at risk" have a strong desire to remain in their home and manage their own care, they often need assistance that is not readily available or affordable; and therefore, often risk more costly and intrusive facility-based care (e.g., repeated hospitalizations, placement in assisted living facilities or nursing homes). Currently, many wellknown and advertised home alert systems directed at aged persons promote reactive approaches to medical emergencies (e.g., summoning help for a fall in the home) rather than being focused on alleviating the infirmities of aging such as dementia/balance and ambulation problems that are often the root causes of declining health and medical emergencies. A change of focus from reactive to proactive home-based monitoring systems to coach behaviors that support wellness (e.g., preventing falls) while responding to real emergencies are sorely needed. One must keep in mind that injuries from falls or conditions that cause unconsciousness (e.g., strokes) render reactive systems useless. In addition, the financial costs of inadvertent 911 calls (false alarms) pose risks to a community with limited care resources. Proactive home monitoring systems that combine the person's medical history along with inexpensive home sensors (motion, pressure. heat\& light) can be used to coach wellness and quickly communicate to family members (or neighbors) significant breaks in daily routines that signal true concerns ("red flags")or "911" emergencies. This paper will discuss and explore options/solutions for improving the daily quality-of-life, health and well-being of medically "at risk" persons that support initiatives for "agingin place" through effective, reliable, low-tech, low-cost, non-intrusive (transparent) electronic home wellness monitoring systems as one possible solution.
\end{abstract}

Keywords: elderly, wellness, home-monitoring, aging in place

\section{BACKGROUND}

This paper will present a health promotion initiative focusing on improving the daily quality of life, health and well-being of medically "at risk" persons. Medically "at risk" persons include elderly (or disabled) persons with one or more chronic diseases, who are living at home[1]. Their numbers are growing with persons over 65 years numbering 39.6 million in 2009 (12.9\% of the U.S. population), to an estimated 72.1 million by 2030 (nearly $20 \%$ of the U.S. population). In 2012, about $28 \%$ of older persons lived alone (8.4 million women, 3.5 million men), and most of these people have one or more chronic conditions such as heart disease, cancer, diabetes, hypertension and arthritis.

Many medically "at risk" individuals with chronic conditions often have difficulty managing their own wellness on a daily basis [2]. They prefer to retain their capacity and effectively manage their own health at home, but often require more costly and intensive facility-based care including recurrent hospitalizations [3]. These individuals have a strong desire to remain in their home and manage their own care, but they often need assistance that is not readily available or affordable. Few practical and affordable healthcare options are accessible for this subgroup. 
Medication noncompliance, poor nutrition and lack of mobility may be positively changed with effective, less-costly, non-intrusive electronic home monitoring. These important causes of poor self-care management are often related to individual behavioral patterns.

Theoretical frameworks: Using the "Hawthorne effect" to promote wellness, it is known that people are creatures of habit and will tend to demonstrate patterns of behavior (i.e., daily habits and routines) over time [4].And, the Hawthorne effect demonstrates that people will change their behaviors if they are aware they are being observed; thus, active monitoring (i.e., via "wellness coaching") may promote more consistent wellness behaviors.

In addition, "coaching" of wellness behaviors through tools such as weekly mailers with patient-centered wellness tips may be part of a consistent, positive reinforcement program. For example, elderly residents in an assisted living facility identified as making consistently poor dietary choices will make improved food choices with prompts, feedback and social reinforcement [5]. Improved results have also been noted with increasing reinforcement of ambulatory exercise using pedometers and providing walking guidelines [6].

These type of guidelines provide a focus on education and simplicity [7]. Elderly patients using medical technology can at times find the technology burdensome as they do not understand or trust the information, and feel that they are somehow limited in lifestyle because of the technology. This results in a barrier in the utilization of technology by elderly residents based on the perception that the technology will not benefit their health. And understandably, the use of technology can also be further constrained by mental and physical infirmities related to aging itself; therefore, ease-of-use in applying technology to help the elderly remain in their homes should be considered.

\section{LITERATURE REVIEW}

Along with elderly persons' inherent desire to remain in their homes, there are many studies that support other constraining factors which indicate the increasing need for the elderly to remain in their homes as well (aging in place). With an increasing elderly population, $85 \%$ of seniors will require some type of in-home care assistance and categories of senior housing (including assisted living and nursing homes) will be challenged to find the right balance of services to attract residents [8]. For each senior house unit available, ten seniors need that space. Less senior housing units are being built each year due to funding levels; however, it is estimated that 730,000 additional senior housing units will be needed by 2020 to address the residential needs of low income seniors.

Long-term care represents a significant financial risk to the elderly in the U.S. and expenditures are unevenly distributed with important implications for the welfare of elderly persons and their adult children [9]. In 2011, the average assisted living rates were $\$ 41,724$ a year and nursing home care costs were $\$ 87,235$ [10]. In 2011, older consumers' out-of-pocket health care expenses averaged \$4,769 showing an increase of $46 \%$ since 2000 [1]. Increasingly strained Federal and State budgets with decreasing subsidies and benefits for long-term care add additional risk to senior citizen health care $[8,11]$.

Our current health care system, including long-term care systems, is not prepared to assist senior citizens with their changing needs [12]. A greater emphasis on self-management strategies where patients learn to manage their own chronic conditions through appropriate exercise and use of medications avoids acute onset of chronic conditions more effectively and slows progression of chronic conditions [13]. The development of naturally occurring retirement communities is an alternative model for aging in a home setting (i.e., "aging in place") [12]. Selfmanagement of chronic illness does not develop as one uniform pattern and wellness activities require support strategies tailored to each person's behavioral development pattern [14].

Increasing high-cost hospital admissions for acute exacerbations of chronic conditions such as congestive heart failure and chronic obstructive pulmonary disease are considered preventable but are often related to inadequate response to outpatient management and home care resources [15]. An additional risk to senior citizen care is an increasing shortage of long-term care workers [16,17]. By 2030, 3.5 million additional long-term care professionals and direct care workers will be needed and there has been a high turnover rate of workers [18].Health care team building can help to offset these losses, including 
restructuring of current practice hierarchy, promoting culture change, providing role clarity for managers, and incentivizing team based care. Geriatric team models could include the following: comprehensive assessment; development and adherence to a comprehensive care plan; proactive monitoring of patients; coordination of care including transitions between hospitals and post-acute care settings and use of community resources [19].
Wireless sensor networks can automate home health care to improve the quality of life,provide early detection of certain conditions, and improve doctorpatient efficiency [20]. Smart home technologies are already available to average users, who would only have a vague awareness of ubiquitous devices but the constant monitoring will increase early detection of adverse conditions for at-risk patients and save lives. Medically "at risk" people have a strong desire to maintain and manage health and wellness in their home versus institutional care [8].

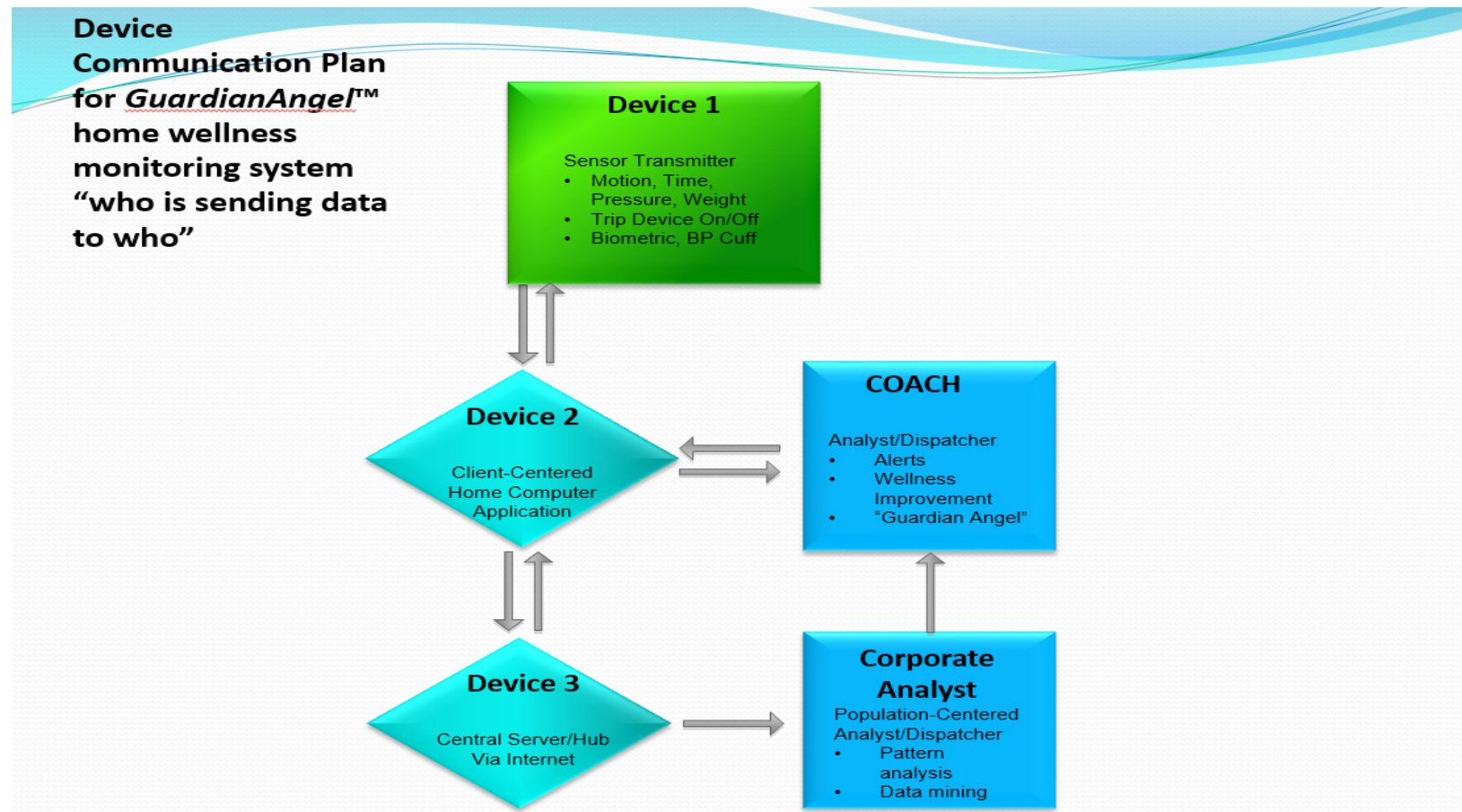

Figure 1. Home Wellness Monitoring System Model. Reprinted with permission from T. Falen, Department of Health Management \& Informatics, University of Central Florida College of Health and Public Affairs, Orlando, Florida.

\section{Discussion}

Developing solutions for medically "at risk" elderly persons to remain in their homes (aging in place) has been a popular discussion of late. The solution we, the authors, propose is the use of low-tech, lowcost, non-intrusive sensors such as motion detectors and biometric sensors. These would be placed in the home to monitor the well-being of medically "atrisk" persons. Wireless sensor transmitters would communicate with a client centered home computer application that would transmit data to a coach using encrypted internet protocols (see - Figure 1). Data analysis would indicate positive or negative behaviors related to well-being. Coaching and interventions will be applied where the person's 1) medical history along with 2) behavioral patterns (i.e., daily routines noted through the sensors) indicate unhealthy behaviors such as medication noncompliance, sitting for long periods of time, and/or instances where significant breaks in patterns (daily routines) occur that may indicate a fall in the home or other medical emergency. Examples of inexpensive home sensors that would be transparent to the user would include those for motion, pressure, light, or heat, etc. They would be placed in a bed or chair for weight and activity; doors, medicine cabinets, cupboards, lights, refrigerators, microwave or television for motion or (body) heat. Depending on the willingness, comfort-level, and abilities of the client, optional interactive sensors would be provided to measure vital signs such as heart rate, pulse, blood pressure, respirations or temperature; and, glucose monitoring as needed. 
Promoting Wellness through Electronic Home-Based Monitoring for Medically "At Risk" Elderly Persons

For a successful outcome, the process would start with an initial client interview to evaluate his or her medical and social history and summary of daily routines. This information would be evaluated for routines of established patterns of a variety of daily living activities (i.e., daily habits and routines). Based on analysis of the findings of the interview, a limited number of sensors (approx. 6) will be strategically placed as "soft" indicators of nutrition (e.g., light sensor that indicates refrigerator was opened), medication compliance, physical activity (e.g., pressure sensor that indicates time sitting in chair), sleep patterns, weight and hygiene (e.g., using motion, light, or pressure sensors). The client would be educated on the technology, its features and benefits. In real time, patterns (and new data) may be evaluated for health alerts and/or behavioral modification recommendations.

The system would be a 24/7 non-intrusive, low-tech, low-cost wellness monitoring system that provides assurance to elderly individuals and their families that they are doing fine. No devices (e.g., necklaces, wristbands, or video streaming) would be worn or managed. The system would conform to the daily routine and would be invisible to the client.

A variety of reminders (e.g., weekly mailer, text, phone call check in) could be sent by a wellness coach to educate and remind the client about the importance of taking his/her medications on schedule, eating a healthy meal, performing exercises to improve balance, etc. This empowers the patient to self-manage to maintain independence. This feedback loop relies on the "Hawthorne effect" to remind patients they are being monitored but in a minimally intrusive manner. The use of coaches builds on a proactive wellness system rather than on a reactive clinical tool. In addition, fewer visits to the emergency room or readmissions to the hospital may result. Client satisfaction would also be an outcome to be evaluated over time. In situations where a significant break in the normal daily routine is sensed (e.g., a client who routinely spends 20 minutes in the bathroom in the morning enters bathroom and does not come out after 1 hour), a notification would be automatically sent to the person's assigned wellness coach. As prescribed by the circumstance (severity of the break in the daily routine), this can result in a series of defined steps that may include 1) coach contacting the client directly 2) notification to an immediate family member or neighbor, or 3) result in a "911" call.

\section{SUMMARY/CONCLUSION}

It is commonly understood, clinicians not only need to focus on a patient's disease process, but spend time getting to know the patient in general and building a relationship with the thought that patients place more trust in people they know and are more apt to comply with their prescribed care[21]. As an analogy, the ability to build a coach-client relationship will promote behaviors that support how elderly individuals may best manage their own care in their own homes. Looking at the bigger picture should improve engagement and lessen dropout rates due to multiple health priorities [22].

According to Healey and Zimmerman [23], a tipping point exists that changes behavior. While the growing numbers of medically "at risk" individuals have a strong desire to remain in their homes and manage their own care, they face losing their homes and independence because of more practical and/or affordable methods to maintain day-to-day wellness that are not readily available in our current health care environment. In a health wellness promotion initiative, success may be the result of changing the home environment, which in turn influences individual behaviors. Less-costly, nonintrusive electronic home monitoring (as opposed to institutional care) to promote wellness for medically at-risk individuals is worthy of further study.

\section{REFERENCES}

[1] Department of Health \& Human Services (2013). Administration on Aging: A profile of older Americans 2012. Retrieved from http:// www.aoa.gov/AoARoot/\%28S\%282ch3qw55 k1qylo45dbihar2u\%29\%29/Aging_Statistics / Profile/2012/14.aspx

[2] Fleming MO, Haney TT. An imperative: Patientcentered care for our aging population. The Ochsner Journal. 2013;13(2):190-193. 
Promoting Wellness through Electronic Home-Based Monitoring for Medically “At Risk" Elderly Persons

[3] Fortinsky RH, Madigan EA, SheehanTJ, TullaiMcGuinness S, Kleppinger, A. Risk factors for hospitalization in a national sample of Medicare home health care patients. Journal of Applied Gerontology, 2012:1-20. Doi:10.1177/0733464812454007

[4] McCarney R, Warner J, Iliffe S, van Haselen R, Griffin M, Fisher P. The Hawthorne effect: a randomized, controlled trial. BMC Medical Research Methodology. 2007:7(30)3 doi:10.1186/1471-2288-7-30

[5] Stock LZ, Milan MA. Improving dietary practices of elderly individuals: the power of prompting, feedback, and social reinforcement. Journal of Applied Behavior Analysis. 1993:26(3):379-387.

[6] Petry NM, Andrade LF, Barry D, Byrne, S. A randomized study of reinforcing ambulatory exercise in older adults. Psychology and Aging.2013:28(4):1164-1173.

[7] Jimison H, Gorman P, Woods S, Nygren P, Walker M, Norris S. Barriers and drivers of health information technologyuse for elderly, chronically ill, and underserved. Agency for Healthcare Research and Quality, Evidence Reports/ Technology Assessments.2008:175(report no. 09-E004).

[8] Society of Certified Senior Advisors. State of the senior housing industry.2013. Retrieved from http://www.csa.us/SeniorHousing Report.aspx

[9] Brown JR, Finkelstein A. Why is the market for long-term care insurance so small? Journal of Public Economics. 2007;91(10):1967-1991.

[10] Gerace A. Broke paying for senior housing \& long-term care? In Senior Housing News, 2012. Retrieved from http://seniorhousingnews. com/2012/02/13/will-the-nation-go-brokepaying-for-senior-housing-long-term-care/

[11] Coggburn JD, Daley DM, Kearney RC. Public sector retiree healthcare benefits: a view from the American states. Public Personnel Management. 2012;41(2):219-240.
[12] GuoKL, Castillo RJ. The U.S. long term care system: development and expansion for naturally occurring retirement communities as an innovative model for aging in place. Ageing International.2012;37(2):210-227.

[13] Ory MG, Ahn S, Jiang L, Lorig K, Ritter P, Laurent D, Whitelaw N. National study of chronic disease self-management: six-month outcome findings. Journal of Aging and Health. 2013;25(7):12581274.

[14] Audulv A. The over time development of chronic illness self-management patterns: a longitudinal qualitative study.BMC Public Health. 2013;13(452):1471-2458.

[15] Kessler R, Faller M, Fourgaut G, Mennecier B, Weitzenblum E. Predictive factors of hospitalization for acute exacerbation in a series of 64 patients with chronic obstructive pulmonary disease. American Journal of Respiratory and Critical Care Medicine. 1999;159(1): 158-164.

[16] Institute of Medicine. Retooling for an aging America: Building the health care workforce. 2008. Retrieved from http://www.iom.edu/ / media/ Files/Report\%20Files/2008/Retoolingfor-an-Aging-America-Building-the Health-CareWorkforce Report Brief Retoolingforan Aging America Buildingthe Health Care Workforce.pdf

[17] Lee W, Dooley KE, Ory MG, Sumaya CV. Meeting the geriatric workforce shortage for long-term care: opinions from the field. Gerontology \&Geriatrics Education. 2013;34(4):354-371.

[18] Eldercare Workforce Alliance. Geriatrics workforce shortage: A looming crisis for our families. 2013. Retrieved from http://www. elercareworkforce.org/research/issue-briefs/ research:geriatrics-workforce-shortage-a -looming-crisis-for-our-families/

[19] Sullivan E, Ibrahim Z, Ellner A, Giesen L. Management lessons for high functioning primary care teams. Journal of Healthcare Management. 2016;61(6):449-465. 
Promoting Wellness through Electronic Home-Based Monitoring for Medically “At Risk” Elderly Persons

[20] Baker CR, Armijo K, Belka S,Benhabib M, Bhargava V, Burkhart N, Der Minassians A. Wireless sensor networks for home health care. Advanced Information Networking and Applications Workshop 21st International Conference 2(2007),832-837.[Online] Retrieved from http://ieeexplore.ieee.org/xpls/abs_ all.jsp?arnumber $=4224209 \& \operatorname{tag}=1$

[21] Schwartz D, Stewart S, Aikens J, Bussell J, Osborn C, Safford M. Seeing the person, not the illness: Promoting diabetes medication adherence through patient centered collaboration. Clinical Diabetes Journal.2017;35(1):35-42.

[22] Fullerton B, Erler A, Pohlmann B, Gerlach, F. Predictors of dropout in the German disease management program for type 2 diabetes. BMC Health Services Research.2012;12(8):doi. org/10.1186/1472-6963-12-8

[23] Healey BJ, Zimmerman RS. The new world of health promotion.2010. Sudbury, MA: Jones and Bartlett

Citation: Thomas Falen, DHSc, MA, RHIA, Alice M. Noblin, PhD, RHIA, CCS, PMP. Promoting Wellness through Electronic Home-Based Monitoring for Medically "At Risk" Elderly Persons. Open Journal of Geriatrics. 2018; 1(1): 1-6.

Copyright: (C) 2018 Thomas Falen, DHSc, MA, RHIA, Alice M. Noblin, PhD, RHIA, CCS, PMP. This is an open access article distributed under the Creative Commons Attribution License, which permits unrestricted use, distribution, and reproduction in any medium, provided the original work is properly cited. 